\title{
Instantaneous fluctuations of temperature and moisture in the upper troposphere and tropopause region. Part 2: Structure functions and intermittency
}

\author{
NiKolai DotZeK* and Klaus Gierens
}

Deutsches Zentrum für Luft- und Raumfahrt (DLR) - Institut für Physik der Atmosphäre, Oberpfaffenhofen, Wessling, Germany

(Manuscript received November 6, 2007; in revised form March 19, 2008; accepted April 21, 2008)

\begin{abstract}
Probability density functions (p.d.f.s) in MOZAIC instantaneous fluctuations of temperature, relative and absolute humidity with various spatial resolutions had shown signs of intermittency. This appears to have originated either from turbulence or certain physical processes (e.g. gravity waves, deep convection) that bring air masses of different origin close together or from air mass boundaries within the averaging volumes, at which the thermodynamical properties show a discontinuity and are nearly independent on both sides of the boundary. In the present paper, we derive a stochastic model for the observed p.d.f.s in the tropopause region and compare the p.d.f.s of the fluctuations in the atmosphere to small-scale Rayleigh-Bénard convection p.d.f.s with similar shapes. In both cases, intermittency originates from rare events of large amplitude superposed unto a Gaussian background. The peculiar ogival shape of some of the distributions results from the sampling procedure, and the ogival character becomes more pronounced with increasing spatial resolution. Analysis of structure functions up to order three reveals that the MOZAIC data represent an intermediate state between large-scale two-dimensional and small-scale three-dimensional inertial range turbulence. This can be visualised by the scale interaction of the typical length of MOZAIC flight legs and the sampling rate (and hence also spacing). While flight legs belong to and exceed the synoptic scale, the events causing intermittency have a scale roughly corresponding to the sampling scale which is clearly sub-synoptic. Our results are directly relevant for the development of stochastic cloud-microphysical schemes in general circulation and weather forecast models.
\end{abstract}

\section{Zusammenfassung}

Wahrscheinlichkeitsdichtefunktionen (p.d.f.s) instantaner Fluktuationen der Temperatur, der relativen und absoluten Feuchte aus MOZAIC-Daten zeigten bei verschiedenen räumlichen Auflösungen Anzeichen von Intermittenz. Diese scheint entweder von Turbulenz oder von bestimmten physikalischen Prozessen (z.B. Schwerewellen, tiefe Konvektion) herzurühren, die Luftmassen verschiedenen Ursprungs nahe zusammen führen, oder von Luftmassengrenzen innerhalb der Mittelungsvolumina, an denen sich die thermodynamischen Eigenschaften sprunghaft ändern, wobei die jeweiligen Werte vor und hinter der Grenze so gut wie unabhängig voneinander sind. In diesem Artikel leiten wir ein stochastisches Modell für die beobachteten p.d.f.s in der Tropopausenregion ab und vergleichen die p.d.f.s der Fluktuationen in der Atmosphäre mit p.d.f.s ähnlicher Form, die bei kleinskaliger Rayleigh-Bénard Konvektion auftreten. In beiden Fällen ergibt sich Intermittenz aus seltenen Ereignissen großer Amplitude, die einem Gauß'schen Hintergrund überlagert sind. Die besondere Ogival-Form einiger Verteilungen resultiert aus dem angewandten Verfahren der Stichprobennahme; sie wird bei zunehmender raumzeitlicher Auflösung stärker ausgeprägt. Eine Analyse der Strukturfunktionen bis zur dritten Ordnung zeigt, dass die MOZAIC-Daten sowohl von großskaliger zweidimensionaler als auch von kleinskaliger dreidimensionaler Turbulenz beeinflusst sind. Dies ist durch die Skaleninteraktion der typischen Länge der MOZAIC Flugstrecken einerseits und der der Abtastrate entsprechenden Strecken andererseits erklärbar. Während Flugstrecken zur synoptischen Skala gehören bzw. diese übersteigen, haben die Intermittenz verursachenden Ereignisse eine Skala, die ungefähr der Abtastdis$\operatorname{tanz}$ entspricht und deutlich unterhalb der synoptischen Skala liegt. Unsere Ergebnisse sind direkt relevant für die Entwicklung von stochastischen wolken-mikrophysikalischen Schemata in allgemeinen Zirkulationsund Wettervorhersagemodellen.

\section{Introduction}

Forthcoming stochastic cloud physics modules in largescale models of the atmosphere (weather forecast and climate models) require knowledge of the probability

\footnotetext{
${ }^{*}$ Corresponding author: Nikolai Dotzek, DLR-Institut für Physik der Atmosphäre, Oberpfaffenhofen, 82234 Wessling, Germany, e-mail: nikolai.dotzek@dlr.de
}

density functions (p.d.f.s) of temperature and moisture in each grid box. In order to assess the characteristics of these p.d.f.s, GIERENS et al. (2007, hereafter Part 1) studied nine years of data from the MOZAIC project (Measurement of ozone, water vapour, carbon monoxide and nitrogen oxides by Airbus in-service aircraft, see MARENCO et al., 1998; BorTZ et al., 2006) and analysed the instantaneous fluctuations that can be ob- 


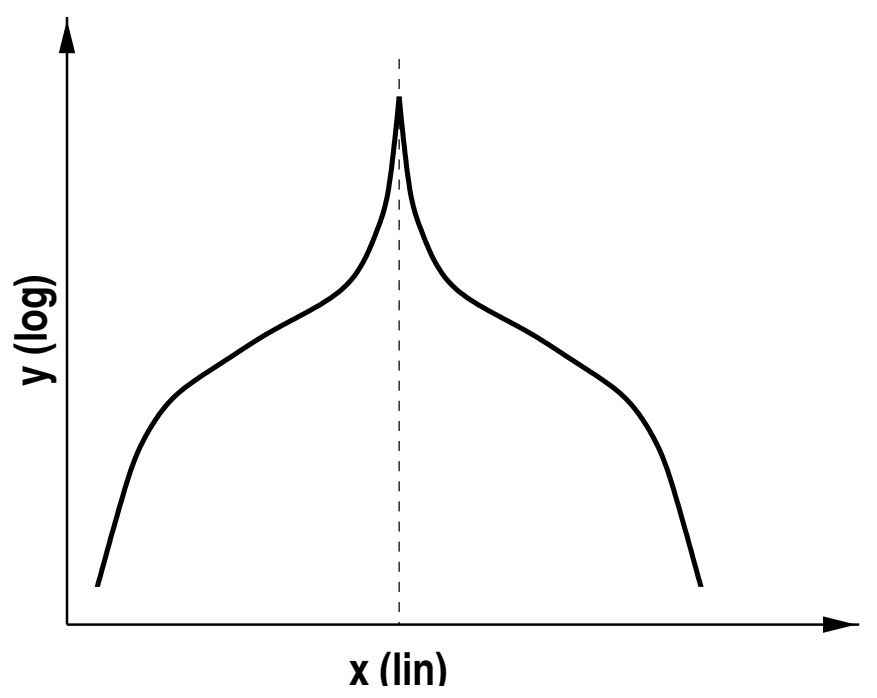

Figure 1: Schematic of a p.d.f. with ogival shape, as classified in Part 1 of our paper, in which such ogival distributions were found both for temperature and humidity fluctuations.

tained from that data base. Within the MOZAIC programme, the large-scale distribution of water vapour has been measured since August 1994 on board of five Airbus A340 aircraft during scheduled flights operated by civil airlines. A major part of MOZAIC data is obtained in the tropopause region during intercontinental flights. Data are available in $4 \mathrm{~s}(\approx 1 \mathrm{~km})$ and $1 \min (\approx 15 \mathrm{~km})$ resolution.

Temperature or moisture fluctuations are called instantaneous if they occur simultaneously within a volume corresponding to a model grid box. Part 1 showed that their p.d.f.s are peaked and clearly non-Gaussian. Whereas the p.d.f. of vapour pressure fluctuations showed convex wings, the two other p.d.f.s displayed a shape named "ogival" in Part 1 and shown schematically in Fig. 1, in particular the p.d.f. of humidity fluctuations. The p.d.f.s of the fluctuations have been determined for various resolutions of a large-scale model, ranging from T21 (grid spacing of about $680 \mathrm{~km}$ at the equator) to T63 (about $208 \mathrm{~km}$ ). The variances of the p.d.f.s decrease with finer grid spacing as a consequence of autocorrelation in the investigated fields. Also the kurtosis values increase strongly with higher resolution, caused by increasingly steeper distribution tails. A Gaussian shape of the p.d.f.s did not appear.

In the present paper, we will investigate the origin of the peculiar shape of the distributions by means of a Monte Carlo simulation. Similarly shaped distributions appear in a variety of fields, e.g. economics (PODOBNIK et al., 2000), spectroscopy (TONKOV et al., 1996), and also in turbulent laboratory flows for which they have been interpreted as signs of intermittency (e.g. Cheng, 1987; Eggers and Grossmann, 1991; She et al., 1991). For the latter, we compare such distributions arising from turbulent Rayleigh-Bénard convection to the MOZAIC data from the tropopause region. Intermittency is a feature of non-linear dynamical systems: Periods of relatively quiescent (regular) behaviour are sometimes, at unpredictable points in time, interrupted by short periods of extreme fluctuations. Intermittent behaviour in the MOZAIC data can also be studied by other means when they are considered as time series, i.e. stochastic processes. Then, the sequence of the data allows to obtain information on autocorrelation lengths, structure functions and scaling behaviour (PIERREHUMBERT, 1996; CHO et al., 2000), or (multifractal) characterisations of non-stationarity and intermittency (e.g. DAVIS et al., 1994; SORNETTE, 2004). These are also topics of the present paper.

Sec. 2 describes the statistical analysis of the scalar fluctuations, focusing on intermittency and structure functions, in relation to analogous formation processes of the MOZAIC p.d.f.s to those of small-scale turbulent Rayleigh-Bénard convection. Sec. 3 discusses mathematical and physical aspects relevant to our analysis, and Sec. 4 presents our conclusions.

\section{Statistical analyses of fluctuations}

There were two remarkable features in the temperature distributions found in Part 1: First, they were peaked at their mean value $(\delta T=0)$ and second, they were by no means Gaussian. Instead, the distribution tails corresponded to exponentials (so-called Laplace distributions) with a slight tendency of an S-shape on both tails of the distributions (i.e. concave-convex on the lower tail, and convex-concave on the upper tail). GIERENS et al. (2007) called this an ogival shape because it resembles certain architectural features of gothic cathedrals, termed ogive.

The distribution of fluctuations of relative humidity with respect to ice, $\delta R H_{i}$, was sharply peaked, nonGaussian and non-exponential, and their ogival shape was very pronounced. It could be explained as the result of a (weighted) convolution of the distributions of $\delta T$ and $\delta e$ (GIERENS et al., 1997), since both fluctuations of temperature $T$ and vapour pressure $e$ contribute to fluctuations of relative humidity.

GIERENS et al. (1997) had used only one year of MOZAIC data and modelled the bivariate p.d.f. of joint fluctuations of temperature and relative humidity by fitting Lorentz distributions (also known as Cauchy distributions) to the 1-d distributions of the fluctuations, but the tails of the distributions in Part 1 differed too much from Lorentzians, so that this approach was not taken again. Instead we will use a Monte Carlo simulation in Sec. 2.1 to evaluate potential causes for the peculiar distributions that we found in Part 1.

Sec. 2.2 will investigate the apparent similarity between the MOZAIC and Monte Carlo p.d.f.s with re- 
spect to those found in small-scale convective turbulence. We then analyse structure functions from the MOZAIC data in Sec. 2.3 and test the tails of the p.d.f.s for presence of stretched exponentials in Sec. 2.4. These mathematical exercises are performed in order to establish the link between the properties of the tropopause region studied here and work of a more general scientific scope in which fluctuations of other quantities (even economic indexes, e.g. PoDOBNIK et al., 2000) are investigated.

\subsection{Ogival p.d.f.s: Monte Carlo simulation approach}

Our concept of the processes that lead to the observed distributions is as follows: Within a certain airmass, a variable fluctuates around a more or less stable basic state. The subsequent fluctuations may be autocorrelated. Intermittently, the basic state changes significantly (e.g. the MOZAIC airplane crosses the tropopause, for other processes see Sec. 3.2), and the fluctuations are now centred around a new basic state. Such a process has been termed Levy-walk or Levy-flight (MANDELBROT, 1983; PodobniK et al., 2000; SornetTe, 2004). We now set up a Monte Carlo simulation in order to investigate which kinds of distributions can arise from such a scenario.

To simulate the random fluctuations, we invoke a discrete Ornstein-Uhlenbeck process (cf. GIERENS et al., 1987; EMBRECHTS et al., 1997): For $n \in \mathbb{N}$ let

$$
\xi_{n}=c \xi_{n-1}+(1-c) Z_{n}
$$

where $Z_{n}$ is a series of mutually independent identically distributed (i.i.d.) increments, and $c \in[0,1]$ is a parameter that controls the correlation between successive values of the process. The expectation value of the increments is zero for all $n$ (different from GIERENS et al., 1987 ), with variance $\sigma_{Z}^{2}$. We choose a standard Gaussian distribution for the increments $Z_{n}$, so $\sigma_{Z}^{2}=1 . \xi_{n}$ is normally distributed for all values of $n$ and all choices of $c$. This follows from complete induction and from the stability of normal distributions. The variance of the $\xi_{n}$ is

$$
\sigma_{\xi_{n}}^{2}=\sigma_{Z}^{2} \Phi_{n}(c)
$$

with $^{1}$

$$
\Phi_{n}(c)=\frac{1-c+2 c^{2 n-1}}{1+c} .
$$

Thus, $\sigma_{\xi_{n}}^{2}$ depends on $n$ (except for $c=1$ where $\sigma_{\xi_{n}}=$ $\left.\sigma_{Z} \forall n\right)$, but it quickly approaches a limiting value

$$
\lim _{n \rightarrow \infty} \sigma_{\xi_{n}}^{2}=\sigma_{Z}^{2} \frac{1-c}{1+c}, \quad c<1
$$

\footnotetext{
${ }^{1}$ A misprint in Eq. (6) of GIERENS et al. (1987) has been corrected.
}

that is independent of $n$. So, the Monte Carlo simulation has a spin-up phase that can be discarded when analysing the results. For $c=0$ we have $\sigma_{\xi_{n}}^{2}=\sigma_{Z}^{2}$, and for $c>0$ the distribution of $\xi_{n}$ is narrower than the distribution of the increments. For large $n$, the variance of the process approaches a constant limit, and that is the reason to prefer this process over a standard Brownian motion process, for instance. The covariance $\varkappa_{\xi_{n, m}}=\sigma_{\xi_{n}}^{2} c^{m-n}$ (for $m \geq n$ ), so that the correlation function

$$
\rho(m-n):=\varkappa_{\xi_{n, m}} / \sigma_{\xi_{n}}^{2}=c^{m-n}
$$

depends in an exponential way on the difference $m-n$, a property that the discrete Ornstein-Uhlenbeck process shares with its continuous counterpart (e.g. SORNETTE, 2004).

We superpose the discrete Ornstein-Uhlenbeck process $\xi_{n}$ onto another process $a_{n}$ (where $n \in \mathbb{N}$ ) representing the intermittently changing background state, i.e. $\xi_{n}+a_{n}$. The process $a_{n}$ is constructed in the following way: We set $a_{1}=0$ and invoke for each $n$ a random number generator giving Gaussian deviates. Only when the selected random number lies in the far wings of the Gaussian (e.g. more than $2 \sigma$ ), the value of $a_{n}$ changes, otherwise $a_{n}=a_{n-1}$. When $a_{n}$ changes, a new value of it is selected at random from a uniform deviate in an interval $[-L,+L]$.

The result of these Monte Carlo simulations is shown in Fig. 2a. The solid line is the resulting fluctuation distribution for a constant background (i.e. $a_{n}=0 \forall n$ ) when the correlation parameter, $c$, is zero, i.e. it is the same distribution as that of the increments, in our case a standard normal distribution. A non-vanishing correlation parameter has the effect to keep the fluctuations closer to the background state, i.e. making the distribution more sharply peaked (as observed in Part 1 for the MOZAIC data). This is shown as the dashed line. A changing background leads to a flat core of the distribution (dotted line).

We can note the following features (cf. MILLER et al., 1995): Without any correlation (i.e. $c=0$ ) and with a constant background, the fluctuation around the background p.d.f. is Gaussian, i.e. of the same type as the increments. When we allow for a 2-point correlation by setting $c$ to a non-zero value, the fluctuation distribution narrows and concentrates around the basic state (here zero). The distribution becomes sharply peaked only for $c \gtrsim 0.9$. When we additionally switch on the intermittently changing background, the distribution becomes flat in its core, which can be perceived as the envelope of a superposition of distributions for a number of different constant background values in the range $[-L,+L]$. The centrally flat distribution is remarkably similar to the distributions of vertical velocity at higher Rayleigh numbers in the direct numerical simulation of 


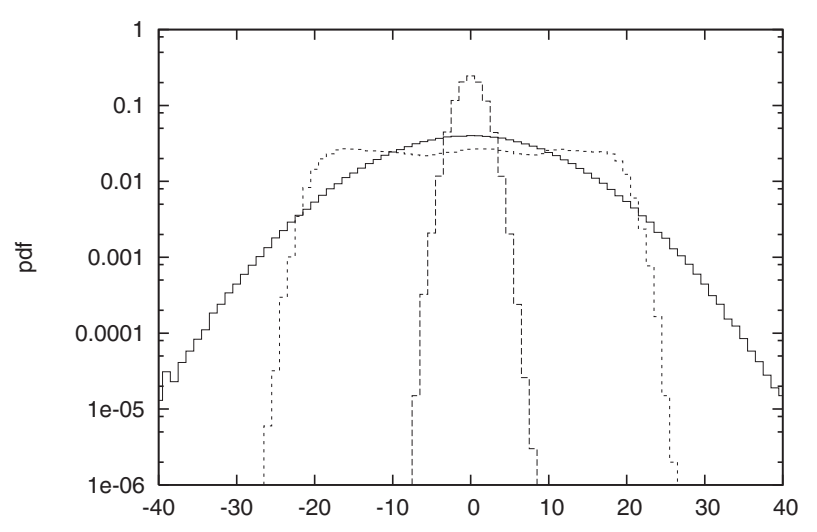

a)

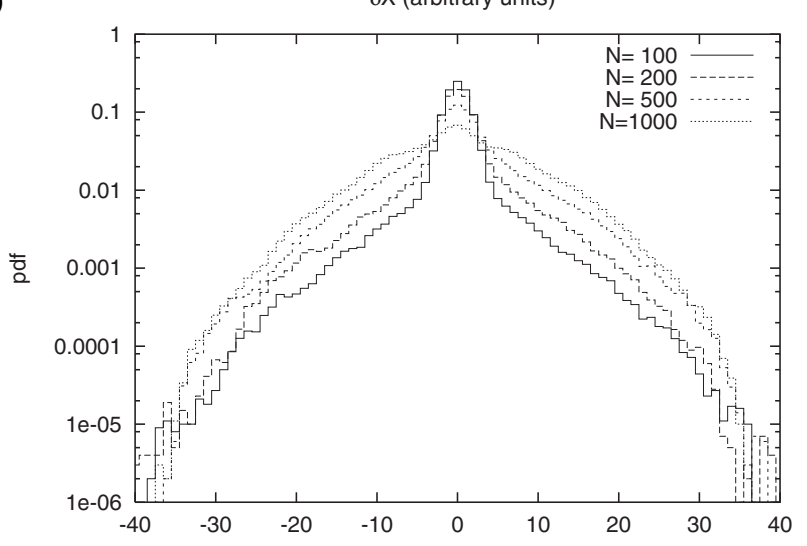

b)

$\delta \mathrm{X}$ (arbitrary units)

Figure 2: P.d.f.s from Monte Carlo simulations: (a) the discrete Ornstein-Uhlenbeck process superposed onto a constant zero background (solid: $c=0$, dashed: $c=0.95$ ) and an intermittently changing background (dotted). In (b), additionally a gridcell-based sampling procedure is simulated. Varying resolution of the underlying grid was simulated with changing the group size $N$, where higher resolution corresponds to smaller group size.

Rayleigh-Bénard convection shown below in Sec. 2.2.

Modeling the ogival shape in the simulations requires an additional step: In our data analysis, we collect all data belonging to the same grid cell, ignoring everything outside for the determination of the fluctuations. We simulate this by conducting a similar sampling procedure. We split our random deviates $a_{n}+\xi_{n}$ in disjoint groups of size $N$, average over each group and record only the deviations of the $a_{n}+\xi_{n}$ to the corresponding group average as the relevant fluctuations. The result of this procedure are the ogival distributions shown in Fig. 2b.

Different group sizes represent different spatial resolutions. As in Part 1 with the MOZAIC data, we see that the distributions are narrowing with higher spatial resolution of the underlying grid, i.e. smaller group sizes $N$.

The Monte Carlo simulation shows that the ogival shape results from the peculiar sampling technique that is necessary when fluctuations within grid cells of large- scale models are to be analysed. Cutting the flight trajectories into pieces that are smaller than synoptic length scales makes it relatively improbable that an airmass boundary occurs in a certain grid box. This together with the autocorrelation in the fields leads to the central peaks in the distributions. The tails, on the contrary, are then formed in those rare grid boxes where an airmass boundary occurs and where hence at least two differing background states are present.

When we set $c=0$ in Eq. (2.1), we obtain again a Gaussian distribution, regardless of changing background and the peculiar sampling procedure. We have also noted the central peak to become more pronounced with increasing resolution. With a group size of $N=10$ (very high resolution, not shown) the central peak protrudes about three orders of magnitude from the distribution tails. Thus, a Gaussian limit distribution cannot be expected for higher resolution.

\subsection{Centrally flat and ogival p.d.f.s in small-scale turbulence}

Interestingly, centrally flat p.d.f.s or such with ogival shape have also been found in direct numerical simulations (DNS) of small-scale thermal convection, namely of Rayleigh-Bénard convection (i.e. convection in a fluid heated from below and confined between two horizontal plates, see KosCHMIEDER, 1993; SHISHKINA and WAGNER, 2006, for an overview). MoENG and RotUNnO (1990) have shown that the Rayleigh-Bénard system can be regarded also as an archetypical representation of atmospheric turbulence, in particular for the unstable planetary boundary layer (cf. WYNGAARD, 1992). Accordingly, KiEMLE et al. (1997) have shown autocovariance functions from near the top of the convective boundary layer which strongly resemble the ogival shape investigated here.

Here, we will analyse the mechanisms leading to the observed p.d.f.s in the Rayleigh-Bénard convection, without making any claim that an analogous phenomenon must exist in the tropopause region. However, we will demonstrate that the processes leading to the ogival or flat p.d.f.s in either small-scale convective turbulence or fluctuations in the tropopause region can be tied to certain key features occurring in both flows. Thus, the results obtained for Rayleigh-Bénard convection will prove to be valuable for identifying the relevant processes for ogival p.d.f.s in the tropospherestratosphere interface region.

Accordingly, we compare results by DoTzEK (1993) and DotZeK and FIEDler (1995) from twodimensional DNS of Rayleigh-Bénard convection in water (Prandtl number $P r=7$ ) with the p.d.f.s from Part 1 and the Monte Carlo simulation in Sec. 2.1 to investigate probable reasons for the observed similarity of the centrally flat or ogival distributions. Fig. 3 shows p.d.f.s 


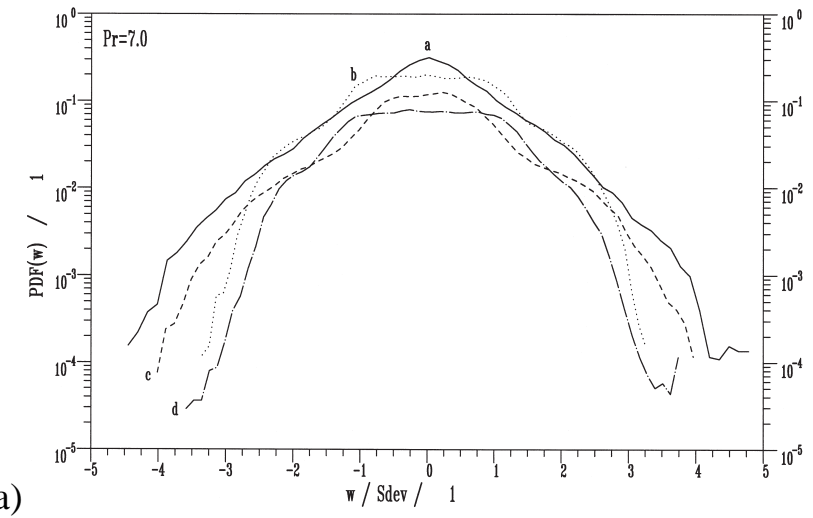

a)

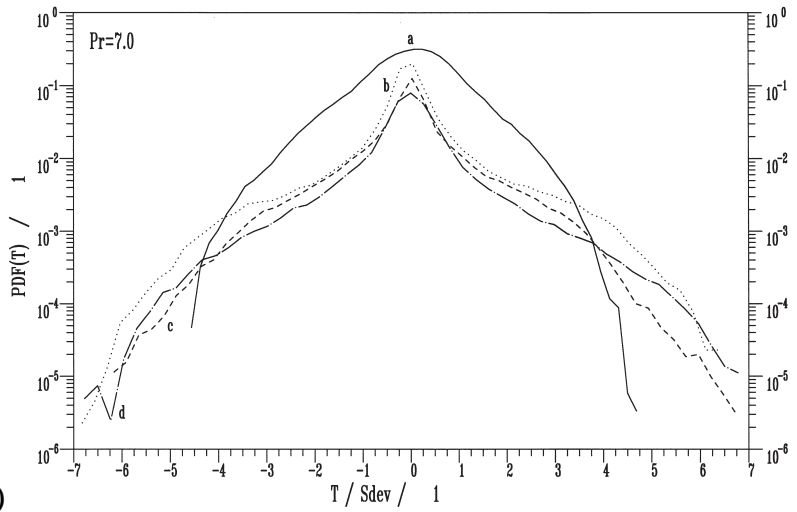

Figure 3: P.d.f.s of (a) vertical velocity, (b) temperature from DNS of Rayleigh-Bénard convection in water $(\mathrm{Pr}=7)$. Fluctuations in $w$ and $T$ have been made dimensionless by their standard deviation $\sigma$. The individual p.d.f.s have been slightly shifted vertically for clarity. Labels at the curves denote: (a) $R a=6 \times 10^{5}$ (including a mean horizontal flow), (b) $R a=7.5 \times 10^{6}$, (c) $R a=10^{7}$, and (d) $R a=5 \times 10^{7}$.

of (a) vertical velocity $w$, and (b) temperature $T$ in the central layer of the convecting fluid with increasing degree of turbulence, expressed by the Rayleigh number $R a$, spanning a range from well inside the soft turbulent to just within the hard turbulent regime (cf. CASTAING et al., 1989; GROSSMANN and LOHSE, 2000; NIEMELA et al., 2000).

The distinction between soft and hard turbulence based on the shape of p.d.f.s has been made by a sudden change from Gaussian to exponential (Laplace) probability densities. The explanation given for this effect is the activity of thermal plumes emanating from the boundary layers. The difference to the case of lower $R a$ is that in the hard turbulent regime, plumes in the middle of the layer are very rare events occurring highly localized in space as well as in time.

Such a flow type would then be likely to produce two states of enhanced probability (cf. LOHSE and Grossmann, 1993; KadAnOFF, 2001): First, due to the strong mixing, it is most likely to find the core region of the fluid in a highly turbulent state, yet close to the volume average of the quantitity observed, e.g. zero vertical velocity or a temperature close to the base-state midplane temperature. Second, the passage of a plume would lead to exceptionally high values of $w$ and $T$ fluctuations. Thus, with increasing degree of buoyancygenerated turbulence, an initially Gaussian p.d.f. will be transformed to one with a high centre peak and heavy tails, i.e. slow decay rates at the sides.

Measurements and simulations first showed that these p.d.f.s could be fit by (stretched) exponentials (Heslot et al., 1987; CASTAing et al., 1989; DeluCA et al., 1990; WERne et al., 1991; ProcaCCIA et al., 1991; Vincent and MeneguzZI, 1991; MASSAIOLI et al., 1993; WERNE, 1993), but this criterion was also doubted (WU and LIBCHABER, 1992; CHRISTIE and DomARADZKI, 1993, 1994; DotZeK, 1993; Siggia, 1994; KERR, 1996), as it became evident that exponentials can be found at low $R a$ also, and there are nonexponential p.d.f.s of a mixed type for cases in which the theory predicted pure exponentials.

Aside from the fact that the p.d.f.s for the lowest Rayleigh number in Fig. 3 are not as symmetric as for the other cases (a non-symmetric mean horizontal flow ${ }^{2}$ was imposed in this case), the p.d.f.s are clearly not Gaussian but show the more or less linear shape characteristic of exponentials. This holds for $w$ in particular and contradicts the assumption that such p.d.f.s can only develop if the turbulence has become intermittent on the way to hard turbulence as described above.

The p.d.f.s of $w$ in Fig. 3a for the higher Rayleigh numbers exhibit a plateau region around $w=0$ which extends to about $1 \sigma$-unit. At $w / \sigma \simeq \pm 2$, another region of slow decrease is located. For larger values of $w / \sigma$, a steep exponential decrease is found. Obviously, these p.d.f.s are neither purely Gaussian nor purely exponential, but of a mixed type. Note the similarity of the central plateau region to that in Fig. 2a. This type of p.d.f. can be understood from the fact that $w$ is not a passive scalar like temperature but a part of the velocity vector. Furthermore, $w$ is directly driven both by buoyancy (i.e. by $T$ ) and by the inertial and pressure gradient forces acting on the convecting fluid confined between the two horizontal plates.

At the lowest Rayleigh number, $w=0$ is the most probable state, and exponential tails indicate the relatively frequent passage of thermal plumes with high vertical velocity deviations from the volume average $w=0$. For the higher $R a$, the flow becomes more turbulent, and just by this turbulence itself, a broader range of fluctuations around $w=0$ becomes more or less equally likely within $\pm 1 \sigma$, even outside the plumes in the bulk of the fluid.

Following a first notable decrease, in particular for $w>1.5 \sigma$, the slower decrease near $w \simeq 2 \sigma$ (the "shoulders" of the distributions) may be due to return flows less

${ }^{2} \mathrm{~A}$ superposition of Couette and Hagen-Poiseuille flow. 
intense than within the plumes, but more intense than the bulk fluctuations. Such return flows may be initiated from a plume impinging on one of the plates and accelerating boundary-layer fluid in the vertical by inducing local pressure gradient forces. For $w>2 \sigma$, steep and roughly exponential tails of the $w$-distributions follow, the characteristic of the passage of thermal plumes with high $w$-values.

A truly ogival shape, however, develops in the temperature p.d.f.s of Fig. $3 b$ from $R a=7.5 \times 10^{6}$ onward. The centre region near zero temperature fluctuation shows a dominant narrow peak of width $\simeq 0.5 \sigma$. For $T / \sigma \gtrsim \pm 3$, curvature has changed, and the p.d.f.s fall off more or less like exponentials. This is again a footprint of the convective plumes, advecting boundarylayer heat and thereby large temperature fluctuations to the midplane region of the fluid layer.

Thus, the temperature p.d.f.s from Rayleigh-Bénard convection have a strikingly similar ogival shape as those from Fig. $2 b$ and from the MOZAIC data of Part 1 obtained on a completely different spatial scale, and a similar argument holds for the centrally flat part of the $w$-distribution compared to those from the discrete Ornstein-Uhlenbeck process. If this similarity is not coincidental, then an analogous mechanism causing it must exist both near the top of the troposphere and in the buoyancy-generated laboratory turbulence.

One candidate process for the atmosphere was the intermittent intersection of discontinuities in the temperature and moisture fields along the flight path, for instance by variations in tropopause height, causing the MOZAIC aircraft to sometimes fly in stratospheric air and sometimes under tropospheric conditions. Other candidate processes are discussed in Sec. 3.2. The result is an intermittent signal in the MOZAIC data of Part 1 as reproduced in the Monte Carlo simulations in Fig. 2.

In the laboratory convection, the exponential or even ogival p.d.f.s result from the intermittency of boundary layer eruptions into the midplane of the fluid layer. Similar to the discrete Ornstein-Uhlenbeck process, the plateau may be envisaged as a superposition of an ensemble of $w$-p.d.f.s shifted by small displacements $\delta w$. Using the purely exponential distribution from curve (a) in Fig. 3a as a basis, we may write the superposition of $2 N$ such distributions as

$$
p_{N}(w)=\frac{\alpha}{2} \frac{\lambda}{2 N} \sum_{i=1}^{N} e^{-\lambda\left|w-w_{i}\right|}+e^{-\lambda\left|w+w_{i}\right|} .
$$

Herein, $w_{i}=(i-1) \delta w$ and $\delta w=2 / N$, for which $N=$ $21, \lambda=4$, and scaling factor $\alpha=0.4$ were chosen as parameters for comparison to the distributions in Fig. 3a. The resulting envelope distributions (not shown) for these and other choices of the parameters revealed that the limit distribution for large $N$ with vanishing $\delta w$ indeed shows a centrally flat region and purely exponential tails. The "shoulders" observed in Fig. 3a are not reproduced thereby, however. This gives us confidence that they do indeed result from a separate physical phenomenon like the return flows mentioned above.

Thus, in the vertical velocity p.d.f.s, the plateau regions can be explained as above by the interaction of a turbulent field with itself (cf. SHE, 1991b). For the temperature p.d.f.s, the situation is qualitatively identical to the MOZAIC humidity data or to Fig. 2b. A quasipassive scalar (temperature in the Rayleigh-Bénard convection, moisture in the MOZAIC data) is superposed to an intermittent field (cf. MiLLER et al., 1995), resulting in the ogival shape of the p.d.f.s. Even a hint of the sampling effect analogous to Fig. 2b can be seen in Fig. 3b: In the discrete Ornstein-Uhlenbeck process, the ogival center peak was most pronounced for the finest sampling. In the DNS of Rayleigh-Bénard convection, the grid sizes were held constant for the simulations shown. However, with increasing Rayleigh number, the coherent structures in the flow become more diminuitive hence the grid size relative to the typical diameter of the structures increases, and the effective sampling becomes coarser. Indeed, there is a tendency for the sharpest peak among the temperature p.d.f.s (b) to (d) in Fig. $3 \mathrm{~b}$ to be found for the lowest Rayleigh number, where the sampling is finest.

Note that the low Rayleigh number and the superimposed mean flow for curves (a) in Fig. 3 prevent both the plateau region in $w$ and the ogival peak in $T$ for the p.d.f.s. Here, the thermal plumes are still broad with relatively weak gradients at their sides. Furthermore, the symmetry-breaking mean flow tilts the plumes, such that a horizontal cross-section runs through the plume in an oblique direction, further reducing the gradients along the horizontal. This underlines the role of steep gradients for the development of intermittency.

\subsection{Structure functions}

Meteorological fields are autocorrelated over certain distances. The sharp peaks in the fluctuation statistics (Part 1) even indicate a rather strong autocorrelation so that a correlation parameter $c$ close to a maximum of unity was required in the simulation to reproduce the observations. In order to obtain more information on the autocorrelation, we computed structure functions and autocovariance functions of the potential temperature for a number of long MOZAIC flights.

Potential temperature $\theta$ is selected to remove any bias from pressure variations on the flights which merely results from altitude changes (the effect of that is but small). We selected the flight with the longest continuous cruise above $275 \mathrm{hPa}$ from each month in 1997. It turned out that with one exception, these were flights between East Asia and Europe. Since the temperature sensor of the MOZAIC equipment is sufficiently fast, 
we take the data in $4 \mathrm{~s}$ resolution, which corresponds approximately to $1 \mathrm{~km}$ distance. The selected flight legs are between 8027 and $10139 \mathrm{~km}$ long.

The autocovariance function of a time series $\xi(t)$ with mean value $\langle\xi\rangle$ is:

$$
\operatorname{Cov}(r)=\langle(\xi(t)-\langle\xi\rangle)(\xi(t+r)-\langle\xi\rangle)\rangle \quad .
$$

$\operatorname{Cov}(0)$ is the variance of the time series, and $R(r):=$ $\operatorname{Cov}(r) / \operatorname{Cov}(0)$ is the autocorrelation function. Evidently, these functions can be perceived as a second moment of the time series. A generalisation to other moments is provided by the structure functions $S_{q}$ :

$$
S_{q}(r)=\left\langle|\xi(t+r)-\xi(t)|^{q}\right\rangle
$$

Obviously there is a close relation between the secondorder structure function $S_{2}$ and the autocovariance function:

$$
S_{2}(r)=2[\operatorname{Cov}(0)-\operatorname{Cov}(r)]
$$

(OBuchow, 1958; Taubenheim, 1969). The main interest in structure functions is to quantify how they scale with lag $r$. For this purpose, one considers relations of the form

$$
S_{q}(r) \propto r^{\zeta_{q}}
$$

and investigates whether $\zeta_{q}$ is proportional to $q$ (normal scaling) or not. If not (anomalous scaling), then $\zeta_{q}$ for $q \neq 2$ contains information that is not already contained in the power spectrum of the time series ( $\mathrm{CHO}$ et al., 2000; DAVIS et al., 1994). Since the covariance vanishes for stochastic processes at lags much larger than the correlation length $r_{c}\left(R \approx e^{-1}\right)$, the limiting value of $S_{2}(\infty)=2 \operatorname{Cov}(0)$. Hence, the power law form of the structure functions can only be valid in a range that does not exceed the correlation length too far. The WienerKhintchin theorem relates the autocorrelation, hence $S_{2}$ and $\zeta_{2}$, with the power spectrum, $E(k) \propto k^{-\beta}$ by their exponents:

$$
\beta=\zeta_{2}+1
$$

First, let us look at the autocorrelation (Fig. 4. On scales that correspond to $\mathrm{T} 42$ resolution of climate models (about $250 \mathrm{~km}$, see Part 1) the autocorrelation typically exceeds 0.5 . Correlation lengths are of the order $1000 \mathrm{~km}$ (i. e. the synoptic scale), which is much larger than the lateral dimensions of climate model grid boxes. Thus, we conclude that typically, there is a pretty high degree of coherence in the potential temperature field within a grid box of a climate model. The same is valid for the temperature itself since the variation of the Exner function $\Pi=\left(p_{0} / p\right)^{R / c_{p}}$ is small, both in our time series as well as from level to level in a climate or weather prediction model. The strong autocorrelation is reflected by the sharp peaks at zero fluctuation in the p.d.f.s of Part 1 ,

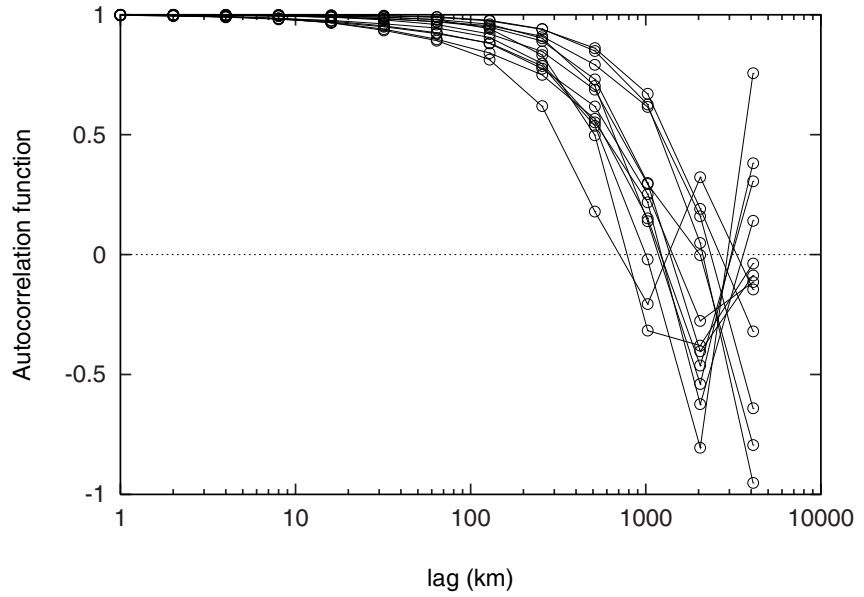

Figure 4: Autocorrelation functions $R$ of potential temperature time series taken from twelve long MOZAIC flights, mainly between Europe and East Asia, in 1997. For each month, one flight has been selected. The twelve curves are plotted together to show the scatter in the data. Lags have been selected as $2^{n} \mathrm{~km}$ with $n \in \mathbb{N}_{0}$ for the determination of $R$ (circles).

and is also consistent with the high correlation parameter required in the Monte Carlo simulation to produce a peak at zero fluctuation.

The second-order structure functions (Fig. 5), computed via the autocovariances, have $\zeta_{2}$ ranging from 0.75 to 1.17 . Accordingly, the exponents $\beta$ in the power spectra range from 1.75 to 2.17 . A similar result with $\beta$ ranging from 1.61 to 2.14 appears when we compute $S_{2}$ directly from the data. The thick line in Fig. 5 represents an empirical fit of the form (cf. MILLER et al., 1995):

$$
\widetilde{S_{2}}(r)=2 \operatorname{Cov}(0)\left\{1-\exp \left[-\left(r / r_{c}\right)^{\zeta_{2}}\right]\right\},
$$

with $\operatorname{Cov}(0)=80 \mathrm{~K}^{2}, r_{c}=1000 \mathrm{~km}$, and $\zeta_{2}=1 . \widetilde{S_{2}}$ has the desired properties for zero and infinite lag (i.e. $\widetilde{S}_{2}(0)=$ 0 and $\widetilde{S_{2}}(\infty)=2 \operatorname{Cov}(0)$. Between $r=0$ and $r=r_{c}$, it behaves like a power law function with exponent $\zeta_{2}$. The corresponding fit to $R(r)$,

$$
\widetilde{R}(r)=\exp \left[-\left(r / r_{c}\right)^{\zeta_{2}}\right],
$$

matches the autocorrelation functions of Fig. 4 well (not shown). The integral scale $L$ in turbulence is associated with the motions containing most of the kinetic energy. It can be computed by integrating the autocorrelation function, i.e. $L=\int_{0}^{\infty} R(r) d r$ (TAUBENHEIM, 1969; LENSCHOW and STANKOV, 1986). If we set $\zeta_{2}=1$ (which is in the range of our data), and insert the expression for $\widetilde{R}(r)$ from above, we find $L=r_{c}=1000 \mathrm{~km}$ which corresponds to the synoptic scale. With $\zeta_{2}=1$, the Fourier transform of $\widetilde{R}(r)$ drops as $k^{-2}$ (i.e. $\beta=2$ ) for wavenumbers in excess of $1 / r_{c}$. In spite of a considerable spread in the structure functions of the individual 
flights, it seems clear that Kolmogorov's classical value of $\beta=5 / 3 \simeq 1.67$ is at the lower end of our range of values. A corresponding fit of $S_{2}$ with $\zeta_{2}=2 / 3$ turns out to be inappropriate for our data. CHO et al. (2000) found $\beta=1.79$ in the extratropical free troposphere for scales from $43 \mathrm{~m}$ to $88 \mathrm{~km}$. This is at the lower limit of our range, but also a bit higher than Kolmogorov's value. It is clear as well that our results do not reach $\beta=3$ which would point to an enstrophy cascade, characterising quasi-two-dimensional "geostrophic" turbulence on the largest, planetary, scales (CHARNEY, 1971; GAGE and NASTROM, 1986). So it seems that our data are affected from both types of turbulence, that is geostrophic turbulence on the largest scales and mesoscale turbulence on the smaller scales.

According to KOLMOGOROV (1958, see also FALKOVICH and SREENIVASAN (2006)), the thirdorder structure function in 3-d turbulence should be proportional to the lag, $r$, for $r$ in the inertial range. Of course, the lags we use to compute the structure functions are so large that the turbulence cannot be considered three-dimensional, and the lags partly even exceed the integral scale. Hence, it is no surprise to find that in our case, $S_{3}$ increases more steeply than linear with $r$, and $S_{3} \propto r^{5 / 4}$ provides a good fit (not shown).

The first-order structure function contains information on the roughness of the graph $g$ of $\theta(t)$ versus time $t$ (see DAVIS et al., 1994). The so-called roughness dimension $D_{g(\theta)}$ is related to the exponent $\zeta_{1}$ :

$$
\zeta_{1}=2-D_{g(\theta)} \geq 0 \text {. }
$$

An almost everywhere differentiable curve has $D_{g}=1$ while a curve that fills a plane has $D_{g}=2$. Our first order structure functions were empirically found to be proportional to $r^{1 / 2}$, that is, $\zeta_{1}=0.5$ and $D_{g(\theta)}=1.5$, lying in the middle of the mentioned extremes. CHO et al. (2000) find smaller values of $\zeta_{1}$, hence their data appear to be less smooth than ours. This is certainly a consequence of their much smaller sampling interval $(50 \mathrm{~ms}$ vs. 4 s). $\zeta_{1}>0$ is an expression of stochastic continuity, that is the difference of two values of $\theta$ becomes small with diminishing lag, as expected. The first three values of $\zeta_{q},\{0.5,1.0,1.25\}$, suggest a non-linear relationship between $\zeta_{q}$ and $q$. This anomalous scaling behaviour is consistent with the results of $\mathrm{CHO}$ et al. (2000) although details differ. The scaling behaviour could be better investigated by considering higher-order structure functions. However, the higher the order, the more sensitive the results are to outliers in the data. We think that only investigation of a much larger number of flights would allow to draw conclusions from higher-order structure functions. Such an investigation is beyond the scope of the present paper.

The relatively low degree of intermittency in the MOZAIC data that is apparent from the low values of

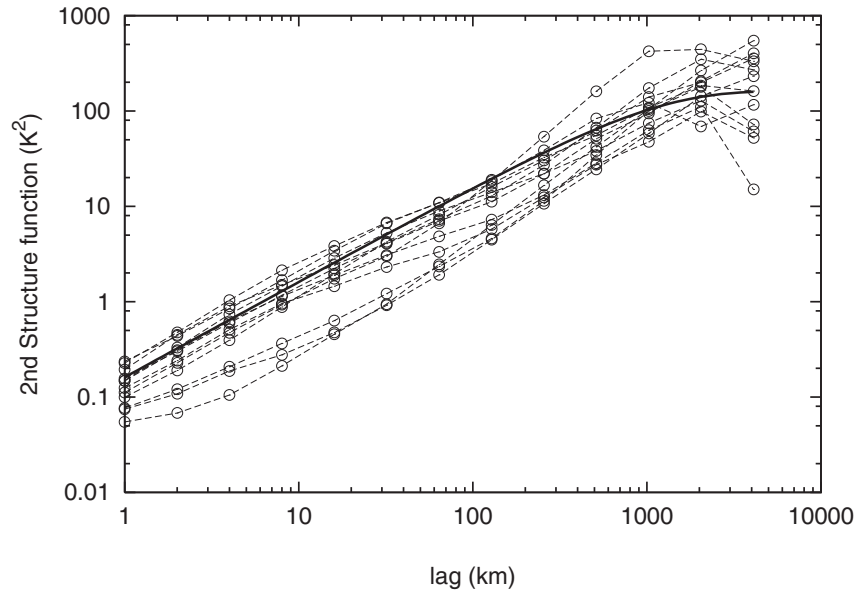

Figure 5: Second order structure functions (dashed lines, circles) of potential temperature time series taken from 12 long MOZAIC flights in 1997, as in Fig. 4. The thick solid line is the fit by Eq. (2.12). Twelve curves are depicted, one for each flight, to illustrate the variability in the data.

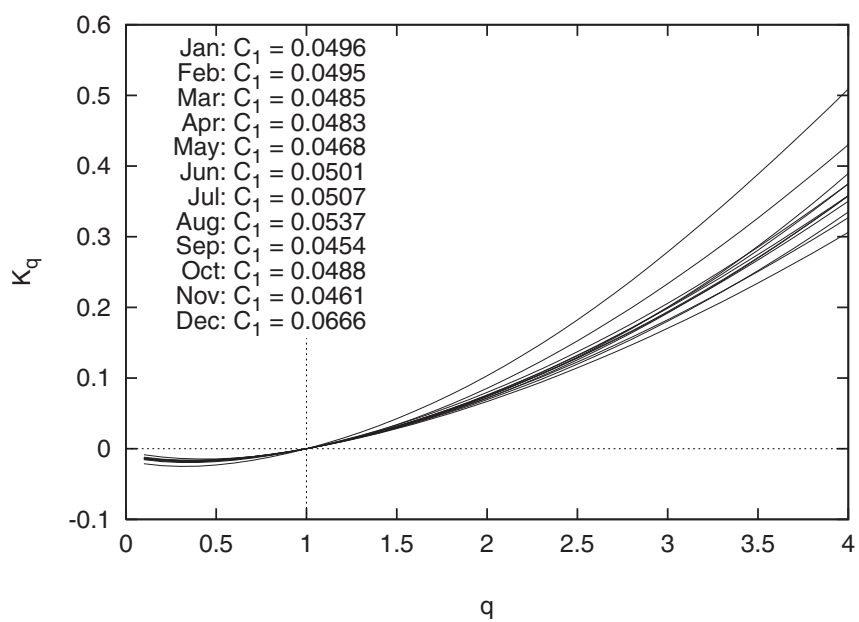

Figure 6: Singularity analysis of scaling exponent $K_{q}$ as a function of $q$, the moment order of analysis, for twelve MOZAIC time series. The intermittency parameter $C_{1}$ is given as the derivative of these curves at $q=1$ and listed in the graph for each month. The ensemble of twelve curves is shown to indicate the degree of variability.

the temperature and humidity fluctuation p.d.f.s in the distribution tails compared to the peaks at zero fluctuation is confirmed by a singularity analysis, for which we follow DAVIs et al. (1994). Their method is related and conceptually similar to the analysis of structure functions, but while the structure functions are made up from differences of the time series over certain lags, the scaling analysis takes the absolute lag-1 differences and averages them in blocks of increasing size, seeking for the largest changes from block to block. This renormalisation-like averaging procedure seems to make the singularity analysis a more robust tool than the analysis of structure functions.

The interest here is how the block averages $\langle\varepsilon(r)\rangle$ and their $q$-th moments $\left\langle\varepsilon(r)^{q}\right\rangle$ scale with block size 
$r$. Similarly to the analysis of structure functions, one makes the ansatz $\left\langle\varepsilon(r)^{q}\right\rangle \propto r^{-K_{q}}$, introducing a hierarchy of exponents. The singularity analysis provides an intermittency parameter, $C_{1}$, and, equivalently, a fractal dimension, $D_{1}=1-C_{1}$ (for details, see DAVIS et al., 1994). The intermittency parameter is small $\left(C_{1} \lesssim 0.1\right)$ for a relatively smooth curve without strong spikes. Fig. 6 shows the hierarchy of exponents $K_{q}$ (corresponding to $\zeta_{q}$ in the structure function approach), obtained from the twelve monthly time series of $\theta(t) . C_{1}$ is the derivative of $K_{q}$ at $q=1$. As expected from the foregoing discussion, we find small values of $C_{1} \simeq 0.05$ $\left(D_{1} \simeq 0.95\right)$ for our twelve time series with one exception where $C_{1} \simeq 0.07\left(D_{1} \simeq 0.93\right)$. These are values typical for smooth curves with low degree of intermittency.

\subsection{Stretched exponentials}

As noted above, the wings of p.d.f.s of fluctuations in turbulence data (measured or simulated) are often described as stretched exponentials (cf. LI and MENEVEAU, 2005):

$$
f(\delta)=\gamma p \delta^{p-1} \exp \left(-\gamma \delta^{p}\right),
$$

with prefactor $\gamma$ and exponent $p$. These stretched exponentials are also known as Weibull distributions and have a wide range of applications (cf. GIERENS and Brinkop, 2002; DotZEK et al., 2003, 2005; FEUERSTEIN et al., 2005). A good method to find the bestfit parameters of a stretched exponential is to plot the data on so-called Weibull probability paper, which is to plot $\log \log \{1 /[1-F(\delta)]\}$ versus $\log \delta$, where $F(\delta)$ is the cumulative distribution function (c.d.f.) of the (onesided) fluctuations. A stretched exponential appears as a straight line in such a plot. We have tested whether the wings of the MOZAIC fluctuations of temperature and relative humidity can well be fit by stretched exponentials. For this purpose, we have averaged the two wings of the corresponding distribution in T42 resolution (Fig. 1 in Part 1), to get a one-sided positive quantity. From this, we computed the c.d.f.s and plotted them on the Weibull probability paper in Fig. 7. We see that humidity fluctuations cannot be modeled with a stretched exponential, which is due to their pronounced ogival character. On the contrary, the temperature fluctuations can be fit very well with a stretched exponential (with exponent $p=0.8$ ).

Stretched exponentials allow for the following interpretation (cf. GIERENS and BRINKOP, 2002): Assume that we measure the (absolute) fluctuation at some point as a function of time. Further assume that the instrument already shows a fluctuation of size $\Delta$. Then we may ask for the probability that the fluctuation size will increase even more, say, to a size $\Delta+\delta$. If the absolute fluctuations were purely exponentially distributed (exponent $p=1$ ), then the answer would be independent

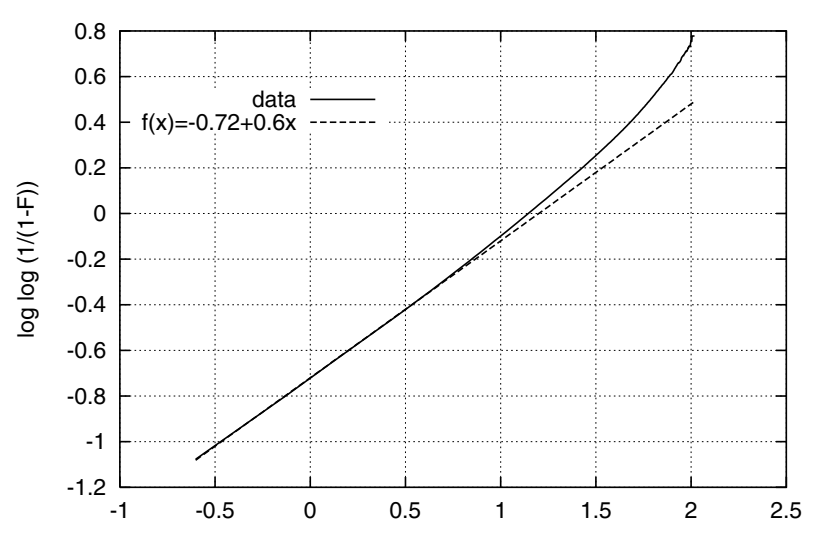

a)

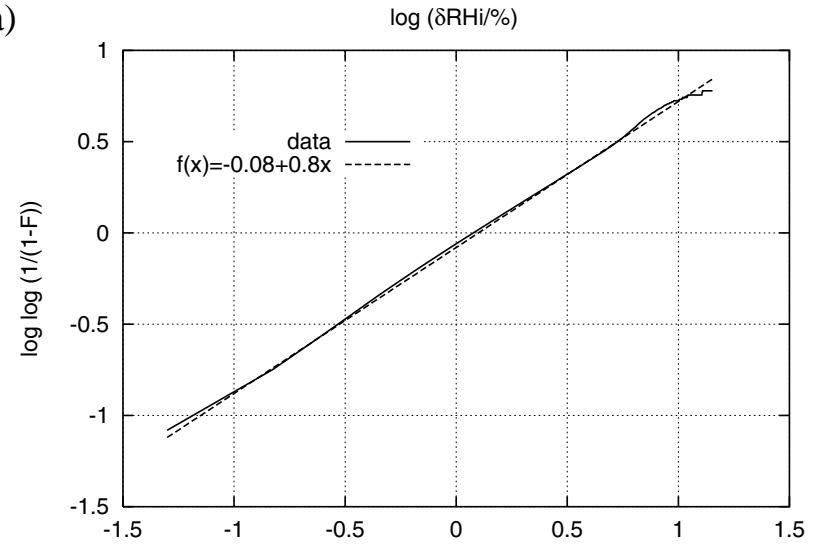

b)

$\log (\delta \mathrm{T} / \mathrm{K})$

Figure 7: Cumulative distribution functions of absolute fluctuations of a) $R H_{i}$ and b) $T$, plotted on Weibull paper, together with linear fits (dashed).

of the value of $\Delta$. In the case of stretched exponentials, however, the probability of an even larger fluctuation increases with $\Delta$. In more mathematical terms: Let $f(\delta \mid \Delta) d \delta$ be the probability that another positive fluctuation between $\delta$ and $\delta+d \delta$ occurs under the condition that a fluctuation of size $\Delta$ is already present, then $f(\delta \mid \Delta) d \delta$ increases with $\Delta$ if $f$ is a stretched exponential.

\section{Discussion}

\subsection{Mathematical aspects - stable distributions and non-Gaussianity}

In our Monte Carlo simulation, we used Gaussian increments to drive the discrete Ornstein-Uhlenbeck process. We showed that this choice implies that the process itself has Gaussian distributions for each $n$. It is of course possible to drive the discrete Ornstein-Uhlenbeck process with increments drawn from another distribution as well. In any case, one should use a stable distribution ${ }^{3}$ for

${ }^{3}$ For the topic of stable distributions see GNEDENKO and KOLMOGOROV (1959), EMBRECHTS et al. (1997), or KUNDU and SIDDANI (2007). 
that. Otherwise, $\xi_{n}$ would have different distributions for different $n$ which would render the model useless. If $Z_{n}$ is drawn from a stable distribution, also $\xi_{n} \forall n$ follow that distribution. Yet, apart from that useful property, all stable distributions except the Gaussian display also strange properties. For instance, they do not have a variance and many of them, for instance the Cauchy distribution, do not even have a mean, because of their fat tails.

The fat tails, on the other hand, might just be appropriate to model variables like our fluctuation data also having tails far above Gaussians. In order to test the utility of other stable distributions, we employed the Cauchy distribution for the increments (centred at zero, full width at half maximum: one). It turned out that the frequent occurrence of values in the far wings of the Cauchy distribution destroys the autocorrelation unless the parameter $c$ has unrealistically high values, e.g. $c>0.9999$. The distributions of the $\xi_{n}$ look equal, apart from some noise for $c=0$ and $c=0.99$. Obviously, the discrete Ornstein-Uhlenbeck process with Cauchydistributed increments is not a useful model of our fluctuation data.

This is also evident from a singularity analysis of the Monte Carlo-produced time series. Whereas the discrete Ornstein-Uhlenbeck process with Gaussian increments shows intermittency parameter and fractal dimension close to those of the MOZAIC data $\left(C_{1} \approx 0.05\right.$, indicating mild intermittency, both with and without changing background), these values differ much when Cauchy-distributed increments are used to drive the discrete Ornstein-Uhlenbeck process. In that case, strong intermittency shows up $\left(C_{1}=0.20\right)$, a lot stronger than in the data. Moreover, the hierarchy of exponents, or the generalized dimensions $D(q)$ for the Gaussian discrete Ornstein-Uhlenbeck process and for the MOZAIC data look similar. Unfortunately, the analytical form of other stable distributions "between" the Cauchy and Gaussian distributions is unknown. Therefore we did not test further stable distributions.

In our analysis of the structure and autocorrelation functions, we found the covariance function to fall off exponentially (using $\zeta_{2}=1$ as above). The discrete Ornstein-Uhlenbeck process had a similar property, $\rho(m-n)=c^{m-n}$. For the data records of the MOZAIC flights, an exponential covariance function implies that two subsequent measurements are autocorrelated with a correlation coefficient $R(\Delta r)=\exp \left(-\Delta r / r_{c}\right)$, see TAUBENHEIM (1969). With $\Delta r=1 \mathrm{~km}$ for the data in $4 \mathrm{~s}$ resolution and $r_{c}=1000 \mathrm{~km}$, this means $R(\Delta r)=$ 0.999. Measurements $n \Delta r$ apart are autocorrelated with a coefficient $R(n \Delta r)=\exp \left(-n \Delta r / r_{c}\right)=R(\Delta r)^{n}$. The MOZAIC data used in Part 1 had 1 min resolution or about $15 \mathrm{~km}$, i.e. $n=15$. Two subsequent data in the $1 \mathrm{~min}$ data are therefore correlated with a coefficient of
0.985, close to the value of $c$ used in our Monte Carlo simulation.

From its construction, it is clear that our discrete Ornstein-Uhlenbeck process is a nonstationary process with stationary increments to be drawn from i.i.d. distributions, and the changing background state makes the process itself non-stationary. The same classification holds for the MOZAIC time series of $\theta$. The analysis of the second-order structure function suggested a value of $\zeta_{2} \approx 1$ which implies an exponent in the energy spectrum of $\beta \approx 2$. In any case, $1<\beta<3$, which is characteristic of non-stationary processes with stationary increments (DAVIS et al., 1994), a property that is common to many geophysical fields.

Several other models have been developed to describe and explain the emergence of non-Gaussian statistics in turbulence, for instance, those by SHE et al. (1988, 1991), SHE (1991a,b), SHE and ORSZAG (1991) or Miller et al. (1995) and Li and Meneveau (2005). These models tackle the problem from a small-scale turbulence point of view, but they also start from acknowledging the observations that

- intermittency is characterised by high-amplitude events embedded in a Gaussian background;

- deviations from Gaussianity become stronger for smaller spatial scales.

The first of these aspects is directly included in our model by the superposition of the discrete OrnsteinUhlenbeck process with the background parameter $a_{n}$ and correlation parameter $c$. The second item is reflected from our explanation of the ogival p.d.f.s from the data sampling characteristics and the fact that finer sampling leads to increasing deviations from the Gaussian shape. Taking into account the sampling procedure as well enables us to explain the ogival shape, while the other mentioned models put their focus only on the (stretched) exponential p.d.f.s in small-scale turbulence. However, our example of the ogival distributions in Rayleigh-Bénard convection is clear evidence that such distributions can arise in (convective) turbulence as well.

\subsection{Physical aspects - potential sources of intermittent fluctuations}

The atmosphere is an open thermodynamic system with highly variable forcing, in particular from solar radiation above and from orographic and topographic effects below. The forcing varies on diurnal, seasonal and longer timescales, as well as geographically due to the relative location of the sun and the non-uniform distribution of land and sea. The atmosphere reacts in a number of large- and small-scale circulation patterns. Additionally, there are atmospheric tides caused by graviational interaction with both sun and moon (CHAPMAN and MALIN, 
1970; ZENG et al., 2008). Consequently, the air is never fully at rest, which always leads to a general background of small variations of the atmospheric state variables in any one place, in particular valid for temperature and relative humidity. However, occasionally larger fluctuations can appear in the state variables. Fluctuations that occur only rarely in space and time are called intermittent. Since we study the fluctuations in larger volumes that correspond to grid cells of large-scale circulation models and not at single locations, the notion "fluctuation" here comprises both local fluctuations caused by a physical mechanism and simple geographical variation within a grid cell, for instance caused by a large-scale gradient or by the presence of an air mass boundary within the cell - which by themselves are consequences of physical processes as well. In the context of intermittency, we call fluctuations arising either from local physical processes or from air mass boundaries (a rare event in a grid box) intermittent, but not the variation arising from a large scale gradient in a grid cell. However, all these variations, whether intermittent or not, must be represented by a large-scale model.

The MOZAIC aircraft (like all commercial aircraft) mostly fly on pressure levels. Hence, flight in baroclinic zones where isotherms are tilted relative to isobars can contribute to large temperature fluctuations (one should say variations in this case, but in the data, the variations cannot be distinguished from the fluctuations). Other sources of temperature fluctuations that exceed the ubiquitous background fluctuations are vertical motions in stably stratified air (i.e. gravity waves, including wavebreaking), and shear instabilities that lead to wave-like displacement of isothermal surfaces, e.g. caused by the Kelvin-Helmholtz instability. Ice-supersaturated regions in the midlatitudes are on average 3-4 K colder than their subsaturated surroundings, while the corresponding temperature contrast is insignificant in the tropics (GIERENS et al., 1999; SPICHTINGER et al., 2003). The average path length of a MOZAIC aircraft through icesupersaturated regions is $150 \mathrm{~km}$, and the aircraft are about $15 \%$ of the flight time in ice-supersaturated regions (GIERENS et al., 1997). Flight into or out of an ice-supersaturated region therefore can cause a substantial jump in the temperature record, causing events in the wings of the p.d.f. of $\delta T$.

Strong fluctuations of relative humidity are also expected when the tropopause is located within a grid cell. Stratospheric air usually is relatively dry, while upper tropospheric air can often be relatively moist, or even ice-supersaturated. On average, aircraft in the North Atlantic flight corridor spend $40 \%$ of their time in the stratosphere (HoInKA et al., 1993). Tropopause folds, where stratospheric air intrudes deeply into the troposphere, occur in association with jet streams (REID and VAUGHAN, 2004). These are strong sources of $\mathrm{RH}_{i}$ fluctuations. Vertical air motions can cause $\mathrm{RH}_{i}$ fluctuations, when air with high absolute humidity is lofted to regions with predominant low absolute humidity, e.g. by deep convection (cf. WANG and SETVÁK, 2007) in environments with neutral or even unstable stratification. In stably stratified air, however, vertical air motions from waves would mainly lead to temperature fluctuations on pressure levels. The presence of clouds in a grid cell is a source of humidity variations (certain microphysical processes only act within clouds) and also for temperature variations (differential radiative heating/cooling and release/consumption of latent heat). The air within icesupersaturated regions (both tropics and midlatitudes) is on average 1.5 to 3 times moister (in absolute humidity units) than in the subsaturated environment (GIERENS et al., 1999; SPICHTINGER et al., 2003). Hence, not only can ice-supersaturated regions produce large temperature fluctuations in the data, but also large humidity fluctuations. Upper level fronts are further boundaries between air masses of different humidity, but the temperature variation across an upper level front is generally weak.

Orographic waves occur mainly connected to hilly or mountainous regions, hence more often in the extratropics than the tropics. Their breaking can cause strong long-lasting turbulence with vertical wind fluctuations of $\pm 1 \mathrm{~m} \mathrm{~s}^{-1}$ (WORTHINGTON, 1998). Such motions can lead to temperature fluctuations in excess of the 1- $\sigma$ values given in Part 1. Assuming a typical lapse rate of about $-5 \mathrm{~K} / \mathrm{km}$, it would only take on the order of minutes for up- or downdrafts with about $1 \mathrm{~m} \mathrm{~s}^{-1}$ to produce a temperature fluctuation that belongs to the wings of the corresponding p.d.f. Increased turbulence due to breaking gravity waves is often confined to shallow atmospheric layers (less than $2 \mathrm{~km}$ ) and in the observations reported by WORTHINGTON (1998), they occurred on 8 out of 76 days, i.e. roughly ten percent of the time. Gravity waves do, however, not always produce fluctuations in excess of the $\sigma$-values of Part 1 . PAVELIN et al. (2002) reported a case of highly localized turbulence (horizontal extent less than $40 \mathrm{~km}$ ) with calculated gravity wave-induced amplitudes of $\pm 0.83 \mathrm{~K}$ for potential temperature (which is just the 1- $\sigma$ value for the temperature fluctuations in Part 1) and of $\pm 0.75 \mathrm{ppm}$ for the water vapour mixing ratio, which corresponds to roughly a $1 \%$ amplitude for $\mathrm{RH}_{i}$ fluctuations (at $-50^{\circ} \mathrm{C}$ and $200 \mathrm{hPa}$ ).

Tropopause folds occur ubiquitously around the jet streams, with their deep specimens even reaching down to the top of the boundary layer (REID and VAUGHAN, 2004). Since commercial aviation from USA to Europe seek the jet stream in order to save fuel and time, it is more likely that these aircraft regularly cross tropopause folds. Not only do the folds represent airmass boundaries that contribute to the larger fluctuations, but they 
also often display turbulence. As the air in the fold is drier than the air underneath, convection can arise from potential instability and cause turbulence extending into the fold. Observations over Wales showed that such turbulence events generally last 1-2 h (REID and VAUGHAN, 2004), while the tropopause folds potentially last much longer (timescale of roughly one day). Tropopause folds certainly are a strong source of humidity fluctuations since the stratospheric air they contain is generally much drier than the tropospheric air in their surroundings.

Clear-air turbulence (CAT, for a recent climatology see JAEGER and SPRENGER, 2007) occurs often, but not exclusively, in the vicinity of the jet streams. Aside from its turbulent velocity field, CAT regions are also candidates for large local fluctuations of temperature and humidity. CAT can be caused by a variety of mechanisms, for instance Kelvin-Helmoltz instability (signified by a low Richarson number) occuring in the strongshear zones of the jets, and symmetric instability (signified by negative potential vorticity) associated with strong curvature of the jet streams. Convective instability (signified by imaginary values of the Brunt-Väisälä frequency) preferably occurs over the continental land masses. All the indicators of CAT show a pronounced annual cycle with minimum number of occurrences (but not necessarily minimum intensity) in the northern summer months. The temperature fluctuation p.d.f. has similar variance in summer as in winter (see Part 1), and $\mathrm{RH}_{i}$ even has stronger fluctuations in summer than in winter. The relative frequencies for various CAT indicators considered by JAEGER and SPRENGER (2007) are on the order of a few percent and the overall frequency of CAT is of the same order, that is, it occurs intermittently.

Summing up the variety of processes that may lead to an intermittent signal for an aircraft flying in the tropopause region, we see that there are three types of intermittency sources:

1. Airmass boundaries, as in particular the tropopause and tropopause folds, but also upper level fronts;

2. Physical mechanisms that bring air masses of different origin close together, for instance deep convection and gravity waves. Ice-supersaturated regions and clouds are consequences of vertical air motions; once they exist they might even enhance the fluctuations already produced by the vertical motion itself;

3. Intense long-lasting turbulence, created by another variety of physical processes like shearinduced instabilities near the jet stream. These may in turn be related to the first two items and can contribute to the measured fluctuations.
Finally, large-scale gradients like in baroclinic zones or caused by orographic forcing from below may be part of the fluctuations that MOZAIC aircraft detect. One could try to investigate the proportion by which each mechanism adds to the intermittent fluctuations or over what geographical regions which process is the most relevant (cf. JAEGER and SPRENGER, 2007), but that would be a topic for a separate paper.

\section{Conclusions}

The present study has led to the following results:

- The peculiar ogival shape of the p.d.f.s of instantaneous humidity fluctuations over volumes corresponding to grid cells of large-scale models can be explained by a combination of autocorrelated fluctuations superposed unto an intermittently changing background state, and the sampling characteristics, i.e. counting and comprising only data that belong to the same grid cell. The intermittently changing background in this model represents traversing airmass boundaries which sometimes occur in grid-cells. These can be introduced by the tropopause itself or by processes like deep convection, gravity waves, and turbulence near the jet stream. The boundary crossings cause the tails of the p.d.f.s. The strong peaks in the distributions signify the strong autocorrelation in the fields.

- The peculiar ogival shape of (passive) scalar p.d.f.s has also been found in studies of turbulence and other phenomena. In turbulence from Rayleigh-Bénard convection, the ogival shape is related to the intermittency of both the velocity and temperature fields. In close analogy to the atmospheric data, there are sharp passive scalar gradients across thermal plumes causing intermittency, and fine spatial sampling being a characteristic of direct numerical simulations.

- The autocorrelation length of the (potential) temperature field, determined from MOZAIC data, is of the order $1000 \mathrm{~km}$, that is, it corresponds to the synoptic scale. On length scales that are typical for the spatial resolution of large-scale models, the autocorrelation exceeds 0.5 . The autocorrelation of subsequent measurements in the MOZAIC time series exceeds 0.95 .

- The power spectrum of the time series of potential temperature has an exponent of $\beta \approx 2$, clearly larger than Kolmogorov's $5 / 3$ and smaller than the $\beta=3$ of geostrophic turbulence. The time series can be considered a non-stationary process with stationary increments. 
- In spite of the higher-than-Gaussian tails of the fluctuation p.d.f.s of Part 1, the intermittency in the MOZAIC time series is weak.

We conclude that a Monte-Carlo simulation as presented here can be used to generate the random deviates that future-generation statistical cloud schemes would need. Development of this generator algorithm, and application of the results to cloud model parameterisations, however, are beyond the scope of this work.

\section{Acknowledgments}

The authors are grateful to Herman G. Smit for kindly providing access to the MOZAIC data and to Christoph Kiemle for his comments on a draft of the manuscript. Insightful suggestions by one of our referees are greatly appreciated. MOZAIC (www.fzjuelich.de/icg/icg-ii/mozaic/) has been sponsored since 1994 by the Commission of the European Communities under Framework Programmes 4, 5, and 6. This work was partly funded by the DLR/HGF-project "Particles and Cirrus Clouds" (PAZI-2).

\section{References}

Bortz, S. E., M. J. Prather, J.-P. CAmmas, V. ThOuRET, H. SMIT, 2006: Ozone, water vapor, and temperature in the upper tropical troposphere: Variations over a decade of MOZAIC measurements. - J. Geophys. Res. 111, D05305, doi:10.1029/2005JD006512.

Castaing, B., G. Gunaratne, F. Heslot, L. P. KadAnOfF, A. Libchaber, S. Thomae, X.-Z. Wu, S. ZALESKI, G. ZANETTI, 1989: Scaling of hard thermal turbulence in Rayleigh-Bénard convection. - J. Fluid Mech. 204, 1-30.

Chapman, S., S. Malin, 1970: Atmospheric tides, thermal and gravitational: Nomenclature, notation and new results. - J. Atmos. Sci. 27, 707-710.

CHARNEY, J. G., 1971: Geostrophic turbulence. - J. Atmos. Sci. 28, 1087-1095.

CHENG, S.-I., 1987: A deterministic view of shear turbulence. - Comp. Meth. Appl. Mech. Engineer. 64, 5-19.

Cho, J. Y. N., R. E. Newell, G. W. SAChSe, 2000: Anomalous scaling of mesoscale tropospheric humidity fluctuations. - Geophys. Res. Lett. 27, 377-370.

Christie, S. L., J. A. DomaradzKI, 1993: Numerical evidence for nonuniversality of the soft/hard turbulence classification for thermal convection. - Phys. Fluids A 5, 412421.

$-,-, 1994:$ Scale dependence of the statistical character of turbulent fluctuations in thermal convection. - Phys. Fluids 6, 1848-1855.

DAVis, A., A. Marshak, W. Wiscombe, R. CAhalan, 1994: Multifractal characterizations of nonstationarity and intermittency in geophysical fields: Observed, retrieved, or simulated. - J. Geophys. Res. 99, 8055-8072.
Deluca, E. E., J. Werne, R. Rosner, F. CAttaNEO, 1990: Numerical simulations of soft and hard turbulence: Preliminary results for two-dimensional convection. - Phys. Rev. Lett. 64, 2370-2373.

DotZeK, N., 1993: Direkte numerische Simulation turbulenter Naturkonvektion in 2D und 3D (Direct numerical simulation of turbulent natural convection in 2-d and 3-d). Diplomarbeit, Inst. Meteor. Klimaforsch. Univ., Karlsruhe, 212 pp. [In German]

DotZeK, N., F. FIEDLER, 1995: Rayleigh-Bénard convection in shear flow. - Preprints, Geophys. Astrophys. Convection Conf. GAC'95, NCAR, Boulder, 45.

DotzeK, N., J. Grieser, H. E. Brooks, 2003: Statistical modeling of tornado intensity distributions. - Atmos. Res. 67-68, 163-187.

DotzeK, N., M. V. Kurgansky, J. Grieser, B. FEuerstein, P. NÉvir, 2005: Observational evidence for exponential tornado intensity distributions over specific kinetic energy. - Geophys. Res. Lett. 32, L24813, doi:10.1029/2005GL024583.

EgGers, J., S. Grossmann, 1991: Does deterministic chaos imply itermittency in fully developed turbulence? Phys. Fluids A 3, 1958-1968.

Embrechts, P., C. KlüPpelberG, T. Mikosch, 1997: Modelling Extremal Events. - Applications of Mathematics 33, Springer, Berlin, 645 pp.

Falkovich, G., K. R. SReEnivasan, 2006: Lessons from hydrodynamic turbulence. - Physics Today 59, 43-49.

Feuerstein, B., N. DotzeK, J. GRIEser, 2005: Assessing a tornado climatology from global tornado intensity distributions. - J. Climate 18, 585-596.

GAGE, K. S., G. D. NASTROM, 1986: Theoretical interpretation of atmospheric wavenumber spectra of wind and temperature observed by commerical aircraft during GASP. J. Atmos. Sci. 43, 729-740.

GIERENS, K., S. BRINKOP, 2002: A model for the horizontal exchange between ice-supersaturated regions and their surrounding area. - Theor. Appl. Climatol. 71, 129-140.

Gierens, K. M., G. TraVing, R. Wehrse, 1987: Formation of resonance lines in media with stochastic depth dependence of the Planck function. - J. Quant. Spectrosc. Radiat. Transfer 37, 361-371.

GiERENS, K. M., U. SCHUMANN, H. G. J. SMIT, M. HeLTEN, G. ZÄNGL, 1997: Determination of humidity and temperature fluctuations based on MOZAIC data and parametrization of persistent contrail coverage for general circulation models. - Ann. Geophys. 15, 1057-1066.

Gierens, K., U. Schumann, M. Helten, H. G. J. Smit, A. MARENCO, 1999: A distribution law for relative humidity in the upper troposphere and lower stratosphere derived from three years of MOZAIC measurements. - Ann. Geophys. 17, 1218-1226.

Gierens, K., R. Kohlhepp, N. Dotzek, H. G. Smit, 2007: Instantaneous fluctuations of temperature and moisture in the upper troposphere and tropopause region. Part 1: Probability densities and their variability. - Meteorol. Z. 16, 221-231.

GNEDENKO, B. W., A. N. Kolmogorov, 1959: Grenzverteilungen von Summen unabhängiger Zufallsgrößen (Limit distributions of sums of independent random variables). - Akademie-Verlag Berlin, 279 pp. [In German.]

Grossmann, S., D. LoHsE, 2000: Scaling in thermal con- 
vection: A unifying theory. - J. Fluid Mech. 407, 27-56.

Heslot, F., B. CAstaing, A. LibChABER, 1987: Transitions to turbulence in helium gas. - Phys. Rev. A 36, 58705873.

HoINKA, K. P., M. E. ReinhardT, W. Metz, 1993: North Atlantic air traffic within the lower stratosphere: Cruising times and corresponding emissions. - J. Geophys. Res. 98, 23,113-23,131.

JAEGer, E. B., M. Sprenger, 2007: A northern hemispheric climatology of indices for clear air turbulence in the tropopause region derived from ERA40 reanalysis data. - J. Geophys. Res. 112, D20106, doi: 10.1029/2006JD008189.

KADANOFF, L. P., 2001: Turbulent heat flow: Structures and scaling. - Phys. Today 54, 34-39.

KERR, R., 1996: Rayleigh number scaling in numerical convection. - J. Fluid Mech. 310, 139-179.

Kiemle, C., G. Ehret, A. GieZ, K. J. DAVis, D. H. LENSCHOW, S. P. ONCLEY, 1997: Estimation of boundary-layer humidity fluxes and statistics from airborne differential absorption lidar (DIAL). - J. Geophys. Res. 102D, 29,189-29,203.

Kolmogorov, A. N., 1958: Die Energiedissipation für lokalisotrope Turbulenz (Energy dissipation in locally isotropic turbulence). - In: Statistische Theorie der Turbulenz, H. Goering (Ed.), Akademie-Verlag Berlin, 77-81. [In German.]

Koschmieder, E. L., 1993: Bénard Cells and Taylor Vortices. - Cambridge Univ. Press, Cambridge, 337 S.

KUndU, P. K., R. K. SidDANI, 2007: A new class of probability distributions for describing the spatial characteristics of area-averaged rainfall. - J. Geophys. Res. 112, D18113, doi:10.1029/2006JD008042.

Lenschow, D. H., B. B. StAnKOV, 1986: Length scales in the convective boundary layer. - J. Atmos. Sci. 43, 11981209.

Li, Y., C. Meneveau, 2005: Origin of non-Gaussian statistics in hydrodynamic turbulence. - Phys. Rev. Lett. 95, 164502.

LOHSE, D., S. GRossmann, 1993: Intermittency in turbulence. - Physica A 194, 519-531.

MANDELBROT, B. B., 1983: The Fractal Geometry of Nature. - W. H. Freeman \& Co. Ltd, New York, 468 pp.

Marenco, A., V. Thouret, P. Nedelec, H. Smit, M. Helten, D. Kley, F. Karcher, P. Simon, K. Law, J. Pyle, G. Poschmann, R. Von Wrede, C. Hume, T. CoOK, 1998: Measurement of ozone and water vapor by Airbus in-service aircraft: The MOZAIC airborne program, An overview. - J. Geophys. Res. 103, 25 631-25 642.

Massaioli, F., B. Benzi, S. SuCCI, 1993: Exponential tails in two-dimensional Rayleigh-Bénard convection. Europhys. Lett. 21, 305-310.

Miller, R. S., F. A. JABeri, C. K. MAdnia, P. Givi, 1995: The structure and the small-scale intermittency of passive scalars in homogeneous turbulence. - J. Sci. Comput. 10, 151-180.

Moeng, C.-H., R. Rotunno, 1990: Vertical-velocity skewness in the buoyancy-driven boundary layer. - J. Atmos. Sci. 47, 1149-1162.

Niemela, J. J., L. Skrbek, K. R. Sreenivasan, R. J. DonNElly, 2000: Turbulent convection at very high Rayleigh numbers. - Nature 404, 837-840.
OBuCHOw, A. M., 1958: Statistische Beschreibung stetiger Felder (Statistical description of continuous fields). - In: Statistische Theorie der Turbulenz, H. Goering (Ed.), Akademie-Verlag Berlin, 1-42. [In German.]

Pavelin, E., J. A. Whiteway, R. Busen, J. Hacker, 2002: Airborne observations of turbulence, mixing, and gravity waves in the tropopause region. - J. Geophys. Res. 107, 4084, doi: 10.1029/2001JD000775.

Pierrehumbert, R. T., 1996: Anomalous scaling of high cloud variability in the tropical Pacific. - Geophys. Res. Lett. 23, 1095-1098.

PoDobnik, B., P. C. Ivanov, Y. Lee, H. E. Stanley, 2000: Scale-invariant truncated Lévy process. - Europhys. Lett. 52(5), 491-497.

Procaccia, I., E. S. C. Ching, P. Constantin, L. P. KadanofF, A. Libchaber, X.-Z. Wu, 1991: Transitions in convective turbulence: The role of thermal plumes. - Phys. Rev. A 44, 8091-8102.

Reid, H. J., G. Vaughan, 2004: Convective mixing in a tropopause fold. - Quart. J. Roy. Meteor. Soc. 130, 11951212.

SHE, Z.-S., 1991a: Physical model of intermittency in turbulence: Near-dissipation-range non-Gaussian statistics. Phys. Rev. Lett. 66(5), 600-603.

—, 1991b: Intermittency and non-Gaussian statistics in turbulence. - Fluid Dynam. Res. 8, 143-158.

ShE, Z.-S., S. A. OrsZAG, 1991: Physical model of intermittency in turbulence: Inertail range non-Gaussian statistics. - Proc. R. Soc. London A434, 101-124.

She, Z.-S., E. JACKSON, S. A. OrsZAG, 1988: Scaledependent intermittency and coherence in turbulence. $-\mathrm{J}$. Sci. Comput. 3, 407-434.

- , 一, 1991: Structure and dynamics of homogeneous turbulence: Models and simulations. - Proc. R. Soc. London A434, 101-124.

SHishKinA, O., C. WAGNER, 2006: Analysis of thermal dissipation rates in turbulent Rayleigh-Bénard convection. - J. Fluid Mechan. 546, 51-60.

SigGiA, E. D., 1994: High Rayleigh number convection. Annu. Rev. Fluid Mech. 26, 137-168.

Sornette, D., 2004: Critical Phenomena in Natural Sciences, 2nd ed. - Springer, Berlin, 528 pp.

SPichtinger, P., K. Gierens, W. ReAD, 2003: The global distribution of ice-supersaturated regions as seen by the Microwave Limb Sounder. - Quart. J. Roy. Meteor. Soc. 129, 3391-3410.

TAubenheim, J., 1969: Statistische Auswertung Geophysikalischer und Meteorologischer Daten (Statistical Evaluation of Geophysical and Meteorological Data). Akademische Verlagsgesellschaft Geest \& Portig K.G., Leipzig 1969, 286 S. [In German.]

Tonkov, M., N. Filippov, V. Bertsev, J. Bouanich, V.-T. Nguyen, C. Brodbeck, J. Hartmann, C. Boulet, F. Thibault, 1996: Measurements and empirical modeling of pure $\mathrm{CO}_{2}$ absorption in the $2.3 \mathrm{mi}-$ cron region at room temperature: Far wings, allowed and collision-induced bands. - Appl. Opt. 35, 4863-4870.

Vincent, A., M. MeneguzzI, 1991: The spatial structure and statistical properties of homogeneous turbulence. - J. Fluid Mech. 225, 1-20.

Wang, P. K., M. Setvák, 2007: Recent new evidences of deep convective vertical transport of water 
vapour through the tropopause. Preprints, 4th European Conf. on Severe Storms, Trieste, Italy, 10-14 September 2007, 2 pp. - [Available from essl.org/ECSS/2007/abs/03Simulation/1177959389.wang-1-sec03.oral.pdf]

WERNE, J., 1993: Structure of hard turbulent convection in two dimensions: Numerical evidence. - Phys. Rev. E 48, 1020-1035.

Werne, J., E. E. Deluca, R. Rosner, F. Cattaneo, 1991: Development of hard turbulent convection in two dimensions: Numerical evidence. - Phys. Rev. Lett. 67, 3519-3522.

WORTHINGTON, R. M., 1998: Tropopausal turbulence caused by the breaking of mountain waves. - J. Atmos.
Solar-Terrest. Phys. 60, 1543-1547.

WU, X.-Z., A. LIBCHABER, 1992: Scaling relations in thermal turbulence: The aspect-ratio dependence. - Phys. Rev. A 45, 842-845.

W YNGAARD, J. C., 1992: Atmospheric turbulence. - Ann. Rev. Fluid Mech. 24, 205-233.

ZENG, Z., W. RANDEL, S. SOKOLOVSKiY, C. Deser, Y. Kuo, M. Hagan, J. Du, W. WARD, 2008: Detection of migrating diurnal tide in the tropical upper troposphere and lower stratosphere using the Challenging Minisatellite Payload radio occultation data. - J. Geophys. Res. 113, D03102, doi:10.1029/2007JD008725. 